\title{
Intervenciones en la seguridad del paciente
}

Interventions in patient safety

Daniela Carrasco Zúñiga,* Fernando Meneses Conzález,*

Miguel Ángel Lezana Fernández*

La revista CONAMED continúa con la premisa de brindar a nuestros lectores información sobre temas actuales y relevantes en temas de salud.

Este número se ha armado con una diversidad de temas que engloban la seguridad del paciente y el cuidado de la salud de la población en general. El primero de ellos es la depresión en cuidadores de pacientes mayores de 60 años, los autores Becerra y Villegas realizan un estudio para conocer la prevalencia en este tipo de población, dividiéndola en depresión leve y moderada y en los sujetos que no la presentan, se cree que en esta población se incrementa el riesgo del deterioro de su salud y su calidad de vida se ve afectada.?

Valle-Rosas y colaboradores presentan los principales factores maternos asociados al abandono de la lactancia materna, la cual es recomendada por la Organización Mundial de la Salud (OMS), durante los primeros seis meses de vida del niño para su desarrollo óptimo.²

La seguridad del paciente como una disciplina en auge engloba el uso de los dispositivos médicos, haciendo uso de la tecnovigilancia como una herramienta que facilita la notificación de incidentes adversos originados por el uso de los dispositivos médicos; Mucito y Sánchez realizan una revisión bibliográfica en donde discuten la importancia de la tecnovigilancia y su instauración dentro de un laboratorio clínico. ${ }^{3}$

Las intervenciones por el personal de enfermería en el cuidado del paciente en ocasiones retrasan la recuperación del paciente. En este número, por el interés clínico en la práctica de enfermería se presenta el abordaje y el análisis de dos casos clínicos, el primero de ellos sobre el manejo inadecuado de injerto en quemadura en un paciente pediátrico, en el segundo caso se analiza la administración de un enema de hipoclorito de sodio, ambos eventos de carácter prevenible por el personal de enfermería, es importante la detección y el análisis de estos eventos para una prevención futura y enseñanza en este campo clínico. 4,5

La sección de seguridad del paciente en enfermería se enriquece en esta edición con recomendaciones en la administración de medicamentos citotóxicos, que por los efectos secundarios que provocan se consideran de alto riesgo al provocar daños a la salud del paciente; la correcta administración y el conocimiento de los efectos secundarios son fundamentales para favorecer la seguridad del paciente. ${ }^{6}$
* Dirección General de Difusión e Investigación. Comisión Nacional de Arbitraje Médico.

Correspondencia: dan.ycz@hotmail.com Conflicto de intereses: Sin conflicto de intereses. Citar como: Carrasco ZD, Meneses GF, Lezana FMÁ. Intervenciones en la seguridad del paciente. Rev CONAMED. 2020; 25(4): 159-160. https:// dx.doi.org/10.35366/97334 Financiamiento: Ninguno.

Recibido: 09/12/2020. Aceptado: 09/12/2020. 


\section{BiblografíA}

1. Becerra-Partida EN, Villegas PCA. Prevalencia de depresión en cuidadores primarios de pacientes mayores de 60 años de edad y con alguna dependencia física. Rev CONAMED. 2020; 25 (4): 161-166.

2. Valle RMP, García TO, Álvarez VAS. Factores que influyen en la madre, en el abandono de la lactancia. Rev CONAMED. 2020; 25 (4): 167-173.

3. Mucito-Varela E, Sánchez-Oviedo FV. Tecnovigilancia en los laboratorios clínicos: una herramienta para la seguridad del paciente. Rev CONAMED. 2020; 25 (4): 174-181.
4. Chanes HD, González-Velázquez MS, Luna D, LezanaFernández MA, Meneses-González F. Manejo inadecuado de injerto en quemadura por parte del personal de enfermería en paciente pediátrico. Rev CONAMED. 2020; 25 (4): 182-187.

5. Molina-Jiménez ME, Ensaldo-Carrasco E, Pineda-Olvera J, Meneses-Conzález F, Lezana-Fernández MÁ. Administration of a sodium hypochlorite enema: a patient safety incident in nursing practic. Rev CONAMED. 2020; 25 (4): 188-192.

6. Romero GDL, Vázquez RA, Del Castillo PÁA. Intervenciones en la administración de medicamentos de alto riesgo: citotóxicos. Rev CONAMED. 2020; 25 (4): 193-196. 